\title{
Management of an acutely disturbed new admission
}

\author{
J. Cookson
}

\section{Case history}

A 25-year-old Bengali man was taken to casualty by relatives who were concerned about his unpredictable and dangerous behaviour. He lived close to his parents and five younger siblings, and there was no family history of mental disorder.

He had been born in Bangladesh and came to the United Kingdom at the age of 12 and completed his schooling. He had worked in the restaurant trade until two years earlier when he left his job after an argument with his employer. He had married at 22; his wife, who spoke little English, was pregnant and they had one son. He had been less regular in his attendance at the Mosque in the previous two years and recently had stopped altogether.

According to his father, he had started to behave strangely two years earlier when he stayed out of his home and neglected his family; he had accused his wife of infidelity. His father had persuaded him to return to Bangladesh where they stayed with an uncle in Sylhet and visited an Imam. They had also attended the psychiatrist at the town's Medical College and he had received injections of fluphenazine decanoate and tablets of trifluoperazine and procyclidine. After four months, he had returned to England, and his family had noticed a marked improvement.

In the past four weeks he had again been staying out from home. He accused his brothers of trying to break up his marriage, and had been violent in his father's home; in a seemingly unprovoked attack he had tried to strangle his brother. He had at times sat motionless for long periods, and at others had appeared over-active, talking excitedly about his plans to take up a college course. His wife reported that she heard him shouting to himself during the night. He said that he had taken tablets obtained from a private general practitioner.

In the casualty department he was examined in a quiet area with a psychiatric nurse from the ward present, in keeping with the hospital's policy for the management of violence. When examined, he sat quietly with a tendency to a fixed gaze and was largely uncommunicative. He appeared tense and preoccupied, but laughed suddenly at times. He was abusive about his brothers, and thought that the doctor was aware of all his personal circumstances: "You know all about me. Don't ask more questions". He expressed plans to study and become a solicitor. He denied hearing voices, and no description of first rank symptoms of schizophrenia was elicited. He said he felt fine and did not consider he had any health problems, but was willing to remain in hospital. He was unable to name the day of the week, but was otherwise orientated.

\section{Physical examination}

An examination showed a tachycardia of 115 , blood pressure $140 / 95$, and a temperature of $38^{\circ}$. His skin was hot and sweaty. He had a moderate degree of rigidity in the upper limbs, but no 'cogwheeling' or tremor.

The provisional assessment was of an acute psychotic illness with suspected auditory hallucinations, persecutory and grandiose ideas and an irritable and elated affect that was rather incongruous. The occurrence of rigidity was noted, and led the admitting doctor to include neuroleptic malignant syndrome (NMS) in the list of diagnostic

Dr John Cookson, FRCPsych, is a consultant and Honorary senior lecturer in psychiatry at the Royal London Hospital, with a catchment area in East London and responsibilities including the Psychiatric Intensive Care Unit, opened in 1988. His research interests are the use of drugs in psychiatry and particularly, the treatment of manic-depressive illness. 
possibilities, which included schizophrenia and bipolar disorder, and catatonia which might also be symptomatic of these. Although the clouding of consciousness and fever might be manifestations of NMS, he was thought to require additional physical investigations to exclude possible infections.

\section{Initial management}

Admission to hospital was necessary for further psychiatric assessment, and to carry out investigations. It was decided to avoid antipsychotic medication until the diagnosis was clearer. He was admitted to a side-room on an open psychiatric ward with a nurse assigned to be with him continuously at first. Referral was made by the hospital psychiatric social worker to the district's Bengali Social Work Team, with a request for them to liaise with the wife and family.

\section{Investigations}

Blood tests were taken for full blood count, urea and electrolytes, liver function tests, creatine kinase, and thyroid function tests, and a urine specimen was obtained for microscopy and culture and for a drug screen. These investigations were all normal with the exception of creatine kinase which was raised at 900 units/1. On the advice of the physicians, an EEG and CAT brain scan were performed and showed no abnormality. It was concluded that he probably had the early stages of NMS, but was otherwise physically well, and that he had a psychotic illness which might include catatonic symptoms.

\section{Further observation}

"As required" medication with oral diazepam 10$20 \mathrm{mg}$ was prescribed to a maximum of $60 \mathrm{mg}$ daily. Further information was to be sought about his recent medication.

On the ward his behaviour was unpredictable and he failed to settle at night. He was given two doses of diazepam $20 \mathrm{mg}$. The following morning he awoke and suddenly seized the nurse who was sitting with him, saying that she was interfering with him. He had to be restrained. His management was reviewed by the doctor and nurses and a request was made that he should be transferred to the Psychiatric Intensive Care Unit (PICU), a small locked ward with a higher staff/ patient ratio, and staff trained in techniques of control and restraint. His temperature had been normal since admission, but his blood pressure fluctuated. Although admission to the PICU did not in itself require compulsory detention, he was made subject to an order under Section 2 of the Mental Health Act (1983) because it had been necessary to restrain him for the protection of other people.

Within the PICU he was initially nursed in his own room, observed by two nurses; diazepam was given orally and he slept for long periods. On waking however he was apprehensive, highly aroused and impulsively violent to those around him. It was thought that he was having auditory hallucinations, but his speech was rambling and fragmented with a paranoid content. He was afebrile and his CK level was lower three days after admission.

\section{Post-admission assessment}

At the post-admission meeting under the Care Programme Approach (CPA), the history and assessment were reviewed. His general practitioner did not attend, but it was confirmed that he had been prescribed trifluoperazine $5 \mathrm{mg}$ t.d.s two weeks prior to admission, and according to his wife he had taken most of this. It was decided that he required antipsychotic medication for the treatment of his psychosis, and that in order to reduce the risk of NMS recurring, phenothiazines should be avoided, and CK and temperature should be monitored. Oral haloperidol was to be given, initially in a dose of $5 \mathrm{mg}$. Procyclidine $5 \mathrm{mg}$ was to be given with each dose of haloperidol to reduce the risk of acute dystonia to which young males in particular are liable.

The meeting identified the community psychiatric nurse who would be involved in the patient's aftercare, and who should be invited to the next CPA meeting. The patient was considered to have a risk factor (serious aggressive behaviour during relapse) which would probably make him suitable for inclusion on the Supervision Register prior to discharge.

\section{Drug treatment}

The junior doctors responsible for urgent decisions about psychotropic medication were aware of the Guidelines for the Pharmacological Management of Violent Psychiatric Patients - a document produced with collaboration between the psychiatric department and the hospital pharmacist. The document summarises the advantages and disadvantages of 
different classes of drugs (antipsychotics, benzodiazepines and amylobarbitone), and different routes of administration (oral, intramuscular, intravenous or depot) in the management of acute behavioural disturbance. It provides detailed advice on doses for individual antipsychotics (chlorpromazine, haloperidol and clopenthixol), benzodiazepines (diazepam, lorazepam and clonazepam), and amylobarbitone. Junior doctors are introduced to this document as part of their induction course on joining the psychiatric department.

In this case, the use of diazepam was favoured by the patient's willingness to accept oral medication (diazepam is less rapidly absorbed by the intramuscular than the oral route); otherwise, lorazepam might have been used intramuscularly (or either diazepam or lorazepam intravenously). The shorter duration of action of lorazepam was considered a disadvantage. Some benzodiazepines are effective in the longer term control of mania (e.g lorazepam in high doses), but others (clonazepam and diazepam) benefit mania and schizophrenia mainly by virtue of their short-term sedative and anxiolytic properties. The choice of antipsychotic lay mainly between haloperidol, clopenthixol and sulpiride. Sulpiride was not favoured because of the lack of parenteral formulations. Haloperidol was preferred as being available by both oral, intravenous and depot formulation; if the patient had consistently refused oral medication after being given an injection (of a benzodiazepine), then clopenthixol would have been preferred because of the availability of the short-acting depot acetate formulation (Acuphase), which can be used to avoid repeated confrontations with reluctant or struggling patients. In this case, in view of the risk of NMS, haloperidol was thought preferable as its duration in the body would be shorter than Acuphase. Haloperidol blocks mainly dopamine but also noradrenaline alpha receptors. Clopenthixol differs from haloperidol also in having a broader spectrum of

Box 1. Discussion points

Differential disgnosis

Role of psychiatric intensive care unit

Rapid tranquillisation; choice of drug and dose

Carbamazepine

Use of Supervision Register

Ethnic aspects pharmacological activity and being more sedative; it is not available for intravenous use.

On commencing antipsychotic medication, the symptoms which were targeted (for monitoring the patient's response) were the apparent auditory hallucinations and unpredictable aggressive behaviour, the incongruous laughter and the abnormalities of speech which made the patient difficult to understand. These positive symptoms tend to improve slowly (from 2-6 weeks) in schizophrenia, but more quickly in mania. In addition, it was expected that the use of an antipsychotic would contribute a more immediate sedative effect reducing hostility and overactivity.

On commencing haloperidol there was no increase in body temperature and no return of muscular rigidity. CK level measured after one and three days showed no elevation. It was therefore decided to increase the dose to $5 \mathrm{mg}$ three times daily together with procyclidine $5 \mathrm{mg}$ three times daily. Two days later, the patient remained unpredictably aggressive and was still being given diazepam up to $60 \mathrm{mg}$ daily. The dose of haloperidol was increased from 15 to $30 \mathrm{mg}$ and later $40 \mathrm{mg}$ daily.

\section{Further progress}

Two weeks after commencing haloperidol, the patient was still in need of close observation, but less p.r.n. diazepam was being used to reduce the risk of his sudden impulsive behaviour. He had established a relationship of trust with nurses and had reported auditory 'command' hallucinations telling him to attack people. He did not feel the nurses were hostile to him. His speech was more normal in speed and less disjointed. A grandiose content remained and his mood was still elated. Full blood count and liver function tests were repeated to detect any idiosyncratic hepatic or bone marrow reaction to the antipsychotic. It was considered whether to increase the dose of haloperidol or to change to another drug. The same dose was continued in order to allow the antischizophrenic effect a full 4-6 weeks to develop.

After six weeks of antipsychotic treatment the patient remained on the PICU, but did not require continuous individual nursing attention. His sleep remained brief and he continued elated and grandiose. He was no longer showing evidence of auditory hallucinations and his speech was less disjointed. In view of the residual manic symptomatology and the tendency to impulsive behaviour he was commenced on carbamazepine. The dose of haloperidol - which might otherwise have been reduced at this time - was maintained, 
because of the tendency of carbamazepine to induce liver enzymes which metabolise other drugs including haloperidol. Treatment with lithium was also considered, but in view of the 'atypical' or schizo-affective pattern of his illness, it was considered less likely to be useful, and the patient was reluctant to have regular blood tests. He and his family were informed of the nature of carbamazepine, the rare risk of serious allergic reactions including agranulocytosis, and the importance of reporting rashes, sore throats or fevers. Blood was to be taken after 2-3 weeks for a blood count, to provide early detection of a delayed hypersensitivity reaction affecting the bone marrow; liver function tests were also to be measured, as were urea and electrolytes to detect hyponatremia.

The dose of carbamazepine was increased gradually from $100 \mathrm{mg}$ to $600 \mathrm{mg}$ daily over the course of two weeks, to reduce early side-effects such as nausea, dizziness, drowsiness and headaches. After two weeks there was further improvement, particularly in the patient's elation, impulsivity and grandiosity; unescorted leave in the hospital grounds was satisfactory, and plans were made for his transfer to an open ward, from where he might begin to have leave from the hospital.

\section{Follow-up}

He continued to improve but was thought to have some residual negative symptoms. Prior to discharge a CPN was introduced to him, and a member of the Bengali Social Work Team was assigned to be his keyworker. He was placed on the Supervision Register. He was advised that he should continue on carbamazepine and haloperidol, with the possibility that depot antipsychotic medication might be more helpful in the future. He returned to live with his wife, and attended out-patients.

\section{Comment}

The case, which is fictitious, is presented not as a model of good practice, but as a vignette to illustrate important decision points that are likely to arise (Box 1).

Management of such a case will vary according to local circumstances which will have brought about development in particular areas, according to prevailing needs and resources. Box 2 lists local policies which may be useful in the management
Box 2. Useful policies in the management of acute behavioural disturbance

Hospital policy on management of violence Intensive care unit: operational policy

Policy on level of nursing observation

Pharmacy policy on prescribing for acutely disturbed patients

Care programme approach: implementation policy

Policy for supervision register

of acutely disturbed patients. The following may merit further discussion or commentary from readers.

\section{Management of violence}

Hospitals should have a written procedure for the prevention and management of violence. There should also be a policy setting out criteria for different levels of nursing supervision, and systems for reviewing and recording the level required. The development of an Intensive Care Unit can benefit the whole hospital by encouraging expertise in the provision of care to patients during acute exacerbations of their illness with less reliance on sedative medication (Hyde \& Harrower-Wilson, 1994). Again a written operational policy is useful to ensure that appropriate patients are admitted. Restraint and seclusion may also be necessary (Fisher, 1994).

\section{Drugs for rapid tranquillisation}

The relative merits of antipsychotics and benzodiazapines were discussed by Dubin (1988). A number of psychiatrists favour a combination of haloperidol and diazepam (Pilowski et al, 1992). The majority of studies of randomised trials of haloperidol in compliant patients have found no advantages, in terms of eventual control of schizophrenic symptoms, in exceeding standard doses (10-20 mg/day), and that very high doses (100-240 mg/day) are associated with more sideeffects and the risk of sudden death (see King, 1994).

For rapid tranquillisation however, higher doses may provide a greater degree of sedation; in this situation the more sedative drugs, such as clopenthixol and chlorpromazine, may be helpful, by virtue of their histamine and alpha-1 receptor blocking effects. The Drug and Therapeutics Bulletin 
(1991) stated that intramuscular chlorpromazine should be avoided as it crystallises in the tissues; this was retracted in the subsequent issue. But chlorpromazine can produce serious hypotension. Droperidol, a drug with potent dopamine and noradrenaline alpha-1 receptor blocking actions, is also liable to lower blood pressure, especially in a dehydrated patient.

\section{High dose antipsychotic medication}

The Royal College of Psychiatrists' Consensus Statement on high dose antipsychotic treatment does not address the use of antipsychotics for acute tranquillisation, but rather the longer-term management of psychosis (Thompson, 1994). However, it is relevant in its recommendation that care must be taken if exceeding the upper limit of doses specified by the British National Formulary (BNF) and in turn in the ABPI Data Sheets. Following a recent change in the Data Sheet for haloperidol, the upper limit for severe or resistant cases of psychosis or dangerously impulsive behaviour in adults is now given as $5 \mathrm{mg}$ three times daily. In adolescents however the dose may be up to $60 \mathrm{mg}$ daily and in resistant schizophrenics the dose may be up to $120 \mathrm{mg}$ daily. In the face of such conflicting information, the clinician must also follow the Consensus Statement's advice that a consultant's opinion should be obtained before the upper limit is exceeded, and a note should be made of the reasons for doing so. The use of very high doses should be a last resort when other means of treatment have failed (Hirsch \& Barnes, 1994).

There is no known pharmacological rationale for the efficacy of high dose antipsychotics (Mackay, 1994), but our understanding of the mechanism of drugs such as clozapine in schizophrenia remains incomplete.

\section{Sudden unexplained deaths}

One of the reasons for caution in the use of medication for the acutely disturbed patient is the number of reports of sudden unexplained deaths in this situation. In some cases, deaths have been associated with high blood levels of antipsychotics (Jusic \& Lader, 1994). However, some cases have involved standard doses of drugs including haloperidol or chlorpromazine, given parenterally sometimes combined with benzodiazepines or amytal. Young men, and those with physical illness (e.g. diabetes) are particularly vulnerable. Cardiac arrhythmias may be responsible in some cases, but in others, respiratory depression (by accumulated doses of barbiturates or benzodiazepines) or laryngeal dystonia causing stridor and hypoxia may be involved.

In the struggling or highly aroused patient, high levels of catecholamines (noradrenaline and adrenaline) which increase myocardial excitability, may be dangerous if the repolarisation process in the heart (due to activation of potassium conductance, and inactivation of calcium conductance) is prolonged. Abnormal repolarisation may be evident in the ECG as a prolonged QT interval or abnormal $\mathrm{T}$ waves. Certain antipsychotics (e.g. pimozide and thioridazine) are known to interact with calcium or calmodulin in the heart to interfere with repolarisation, causing prolongation of the $\mathrm{QT}$ interval and abnormal $T$ waves. Many other antipsychotics (phenothiazines and butyrophenones, but less so benzamides and thioxanthines) are known to have membrane-stabilising effects at very high doses. These effects (also known as quinidine-like, local anaesthetic, or type- 1 antiarrhythmic) involve blockade of sodium channels. In this regard, the antipsychotics resemble tricyclic antidepressants and would be expected to cause atrioventricular or intra-ventricular conduction delays including AV block or bundle branch block.

It remains unclear how standard doses of these antipsychotics might interact with states of excitement or exhaustion to produce fatal arrhythmias. When high doses of antipsychotics are used, or if the intravenous or parenteral route is used for the purpose of sedation, the patient should not be left unattended. The nurse assigned to them should check and record the pulse, blood pressure and respiration and should be sufficiently trained to recognise symptoms of respiratory depression, dystonia or dysrrhythmia. In case of respiratory depression, they should be able to provide oxygen through a mask or an Ambu bag, and be aware of the need to alert medical staff.

\section{Initiating carbamazepine}

Care is required in the use of carbamazepine because of the risk of allergic reactions. The current Data Sheet recommends that blood tests be carried out before starting treatment and periodically during treatment, but that clinical monitoring is of primary importance. The earlier Data Sheets had advised more frequent blood counts. Although the license extends only to the prophylaxis of lithiumresistant manic-depressive psychosis, the drug is also useful in combination with haloperidol in excited psychotic patients (Klein et al, 1984). 


\section{Conclusion}

This case illustrates a number of decision points (see Box 1). Readers may wish to comment on these or other matters raised by the case. APT invites discussion of controversial issues.

\section{References}

Drug and Therapeutics Bulletin (1991) Management of behavioural emergencies. Drug and Therapeutics Bulletin, 29, 62-64.

Dubin, W. R. (1988) Rapid tranquillisation: antipsychotic or benzodiazepines? Journal of Clinical Psychiatry, 49 (12, suppl), 5-11.
Fisher, W. A. (1994) Restraint and seclusion: a review of the literature. American Journal of Psychiatry, 151, 1584-1591.

Hirsch, S. R. \& Barnes, T. R. E. (1994) Clinical use of high-dose neuroleptics. British Journal of Psychiatry, 164, 94-96.

Hyde, C. E. \& Harrower-Wilson, C. (1994) Psychiatric intensive care: principles and problems. Hospital Update, 287-295.

Jusic, N. \& Lader, M. (1994) Post-mortem antipsychotic drug concentrations and unexplained deaths. British Journal of Psychiatry, 165, 787-791.

King, D. J. (1994) The use of high doses of neuroleptics: the current situation. International Clinical Psychopharmacology, 9, 75-78.

Klein, E., Bental, E., Lerer, B., et al (1984) Carbamazapine and haloperidol $v$. placebo and haloperidol in excited psychoses. Archives of General Psychiatry, 41, 165-170.

Pilowski, L. S., Ring, H., Shine, H. G., et al (1992) Rapid tranquillisation. A survey of emergency prescribing in a general psychiatric hospital. British Journal of Psychiatry, 160, 831-835.

Thompson, C. (1994) The use of high dose antipsychotic medication. British Journal of Psychiatry, 164, 448-458.

Mackay, A. V. P. (1994) High dose antipsychotic medication. Advances in Psychiatric Treatment, 1, 16-23. 